\title{
The Application of Virtual Reality Technology in Library Information Retrieval
}

\author{
Wei Luo \\ Zhuhai College of Jilin University \\ Zhuhai, China
}

\begin{abstract}
The rapid development of virtual reality technology has penetrated into the culture and education industry. It has also brought challenges and opportunities to the university library. Based on the definition of virtual reality technology and its application in domestic and foreign university libraries, this paper analyzes and researches the application of virtual reality technology in library information retrieval. The extensive promotion and application of virtual reality technology can provide readers with a visual, interactive and perceptive digital library to achieve effective integration of virtual library and physical library, enhance information retrieval service function and service capacity and maximum the value of literature resources in library.
\end{abstract}

Keywords-university library; virtual reality; information retrieval

\section{INTRODUCTION}

In recent years, with rapid development of the digital age, the traditional paper-based documents in library have been increasingly impacted by diverse information terminals. At the same time the paper documents and collections are also more and more limited by the development of space. The traditional library has been unable to satisfy the needs of readers, so the university library, as the cultural information center, shall adapt to the development needs of the Internet age. It is necessary to reasonably allocate paper resources and electronic resources, adjust resource structure and gradually transform traditional paper-based mode into digital mode.

As the infrastructure of the information age, digital library develops rapidly. Meanwhile, there are also some problems. First, the digital library is not intuitive. At present, the vast majority are two-dimensional and planar. They lack stereo perception, are not visualized enough and with no space atmosphere. Second, the printed documents in traditional library are not suitable for network transmission. Although many readers love to read paper literatures, it is inconvenient to carry. Third, as for service in the digital library, it is unable to realize the visualization in current information retrieval process and result. It cannot provide comprehensive and intuitive knowledge structure association and lacks interactivity. And virtual reality technology has characteristics of multi-perception, visualization, immersion,

[CLC] G250. interactivity and imagination.

The application of virtual reality technology to digital library can easily solve the problems of lack of visualization and interaction in digital library. Therefore, this paper will study and explore the application of virtual reality technology in library literature retrieval. The application of the technology in the construction and retrieval of the library literatures can provide the reader with a visual, interactive and perceptive digital library. It can also realize the effective integration of virtual library and physical library, enhance the service function and service capabilities of information retrieval and maximum the value of literature resources in library.

\section{VIRTUAL REALITY TECHNOLOGY}

Virtual reality technology (hereinafter referred to as VR) is a computer simulation system that can create and experience a virtual world. It creates a simulation environment with computer. It is a simulation system with multi-source information fusion, interactive 3D dynamic view and entity behavior, which enables user immerse in the environment.

VR mainly includes simulation environment, perception, natural skills and sensing equipment. Besides the threedimensional realistic images generated by computer graphics, there are various perceptions, including hearing, touch, force, motion, and even olfaction and taste. The body actions of human is processed by computer to form corresponding data, making real-time response, and then feedback back to user's facial features, giving users immersive experience, and not limited by distance and space. VR has the characteristics of simplicity, strong interaction ability, and suitable for low bandwidth transmission. It has been widely applied in many industries, such as industrial transportation, medical devices, education and training, aerospace and real estate development.

\section{THE APPLICATION OF VR IN DOMESTIC AND FOREIGN UNIVERSITY LIBRARIES}

\section{A. The Application of VR in Foreign University Libraries}

The investigation of the libraries in the 2015 Top-fifty World Universities according to QS, in Comparative Study of Application of Virtual Reality in University Library at 
Home and Abroad, shows that 48 foreign universities are using virtual reality except for Tsinghua University and Peking University. At present, 11 university libraries provide service with virtual reality in their websites, including the libraries in National University of Singapore, University of Oxford, St Edmund Hall and Harvard University.

\section{B. Application of VR in Domestic University Libraries}

- The national library is the first one to use virtual reality system in China: In September 2008, the virtual reality system was officially launched in our national library. It is the first set of interactive virtual digital library system in China. It marks the start of the use of VR in the domestic library.

- Zoomii virtual bookstore on the Internet realizes 3D browsing: In June 2008, a company called zoomii online bookstore officially released. Users can enter into the virtual bookstore through clicking the mouse, and they seem to be in the physical bookstore personally. Books are displayed in accordance with the classification. Use the mouse to zoom, move around. It seems to be in the physical bookstore. Users can also click on the book to leaf through and then decide whether to buy.

- According to the survey results of relevant references, a total of eight colleges and universities are using the virtual reality in their libraries, including Tsinghua University, Wuhan University, Zhongshan University, Huazhong University of Science and Technology and University of Science and Technology of China among top 50 colleges and universities in China. At the same time, some colleges and universities are promoting and testing the VR.

After occupying two major fields, game and entertainment, VR has launched an attack on the fields of culture and education. Various colleges and universities have paid attention to the application of virtual reality to their digital libraries.

\section{THE APPliCATION AND RESEARCH OF VIRTUAL REALITY IN INFORMATION RETRIEVAL SERVICE}

As an interdisciplinary subject, virtual reality technology combines many new and high technologies. Its three main characteristics, immersion, interaction and imagination, have penetrated into all walks of life. With big impact of the network era, university library shall keep pace with the times, change the traditional paper-based literature mode, reasonably change the proportion of digital resources and form, and fully apply VR and other new technologies into information retrieval services. It can provide strong technical support in teaching, scientific research and social service in colleges and universities, and improve the service level of the library, innovate and increase the variety of service, provide a new, fast and intuitive service experience for readers, and eliminate the limit in space and distance in library.

\section{A. Use VR to Realize the Space Navigation and Roaming of the Library, and Provide Readers with the Guide Reading Function}

The purpose of guide reading in library is to cultivate readers' reading consciousness, reading skills and reading methods, help master methods of document information retrieval, and improve readers' interest in reading. The guidance provided by the traditional library is nothing but entrance education, the literature retrieval course, and the similar trainings, etc. The use of VR can realize real shooting on layout of internal space and facilities outside library, providing readers a 360 -degree virtual roaming space. The use of 3D modeling way enables resources in library display in 3D environment. The VR guide system can eliminate limit in space and region. Readers seem to be personally in the library. Readers can see layout of all function areas in library, classification of literatures and collections and relevant services by virtual roaming. It can maximally meet reader's needs in the shortest time.

\section{B. Use VR for the Construction of Digital Resources}

The information resources in university libraries are varied and colorful, including paper books, audios, videos, database and so on. But the traditional paper books can only be displayed in a two-dimensional way. Some special information resources, such as ancient and precious historical documents, cannot be fully displayed in front of the readers because of the limitations, which limit the information transmission effect. However, the use of virtual reality can transform these precious historical documents and resources of special subjects into three-dimensional virtual resources. It enables readers read or appreciate these valuable information resources. In addition, it is also conducive to the preservation and research of resources.

For example, the digital protection of cultural relics is an important means for the permanent preservation and sustainable utilization of cultural heritage. On May 1, 2016, the Dunhuang Institute for the first time officially launched the high-definition digital contents and panoramic roaming of 30 classic grottoes in Dunhuang Grottoes. The resource platform of "Digital Dunhuang" has been using modern digital technology to shoot, scan, obtain and store relic information of Dunhuang Grottoes, establishing a diversified and integrated database of the digital Dunhuang, a digital asset management system and a permanent preservation system of digital resources. It has realized the permanent preservation of Dunhuang culture and art resources, providing endless possibilities for academic research and realizing the multi-use of the resources. Therefore, the application of VR in the construction of data resources can achieve permanent preservation of previous literatures and cultural relics, breaking through the geographical restrictions. It enables more readers to access to. Then these resources can be continuously and effective used in education, art, tourism and other fields, and play a greater academic value and art value. 


\section{Use VR to Integrate Data Resources Reasonably}

Colleges and universities generally have a huge discipline system, involving a wide range of disciplines. Library is serving for extensive teaching and scientific research, so it needs a large number of systematic paperbased or digital resources to support and serve. The use of VR can classify resources of various disciplines, especially cross-disciplinary resources, and establish connections. When readers search relevant literature resources of interdisciplinary, it helps them avoid going to different function areas and search different interfaces, and reduce a lot of time and energy. If searching digital resources, readers can download APP and browse related resources without convert reader or related software. All resources can be access to.

\section{Use VR to Provide Literature Retrieval Service for Readers}

After referring to relevant information, it found that currently three domestic universities are using VR to provide information retrieval services for readers in library. Nanjing Normal University has real-time virtual bookshelf in library. Shanghai Jiao Tong University and Nankai University have positioning and navigation system in their libraries. Among them, the book positioning and navigation system in library of Nankai University is still in the stage of test and check before acceptance.

The virtual reality can provide a real-time virtual bookshelf for readers. In the retrieval of books, readers can see a virtual bookshelf. Through the system, readers can see the retrieved book and books on its left and right sides, and browse more book information through the control buttons.

The book positioning system is a positioning operation with the application of VR based on the RFID book positioning system. When readers retrieve a book, the RFID system on OPAC will show the specific position of the book by words. Click the label of "book positioning", and then the VR will show the 3D virtual environment and specific location of the book, so that readers can quickly and intuitively find the required documents. However, at present, there are still some difficulties in implementing VR retrieval services, which still need to be further refined and explored, so as to achieve popularization and wider application.

\section{E. The Use of VR Can Provide Readers with Personalized Reference Services and Characteristic Reading Experience}

The panoramic mode presented by the virtual reality technology is with a strong sense of reality. It helps readers be familiar with the layout of the library and exterior buildings as soon as possible. It helps readers reasonably optimizes the path, get the resources needed in time and efficiently, and reduce the waste of time and space. At the same time, it provides reference service for readers. Readers can get virtual roaming of real scene and have experience with real feeling.

\section{F. Use VR to Better Carry out the Entrance Education for Freshmen}

Use VR to carry out entrance education for freshmen. It enables students to bid farewell to the traditional boring course. The virtual roaming of real scene can help the freshmen receive information and bring them sensory experience. Library is apparent to eye with VR, which can attract and improve reading interest of readers.

\section{CONCLUSION}

In the digital age, university libraries are constantly impacted by the rapid development of the Internet era. Virtual reality is bound to bring unprecedented experience and impact to all aspects of university libraries. With the application of VR, high-quality, humanized and characteristic services can be provided to readers in literature retrieval, information resources construction and service innovation. Read thousands of books, and do not need to travel thousands of miles. The use of network can integrate the information resources and improve the knowledge system of readers, so that readers can enjoy the reading experience that has never had before. It can help attract more readers to participate in reading. We should take a positive attitude to focus on and promote the development of VR, effectively locate the application of VR in university library, and effectively integrate it into the cause of university library, and promote the continuous development of university library cause.

\section{REFERENCES}

[1] Qian Li, Zhang Zhixiong, Zou Yimin \& Huang Yongwen. The Application of Information Visualization Retrieval in Digital Library. Modern Library and Information Technology, 2012 (4).

[2] Wei Meng, Wang Yingchun \& Liu Yanquan. A Comparative Study on the Current Situation of Smart Services in the Libraries of China and the United States. Research on Library Science, 2017 (19).

[3] Wang Hong, Wang Huitao. Research on the Construction of 3D Virtual Library in Colleges and Universities under the Game Trend. Modern Information, Mar. 2017 (37-3).

[4] Zhou Lihong, Han Yingying \& Tu Xiaomei. Comparative Study on the Application of Virtual Reality Technology in University Libraries at Home and Abroad. Books and Information. 2017 (4). 